\title{
Mechanistic pathway of water adsorption to impact on the nonlinear
} elasticity of single-crystalline ZnO NWs

\author{
R.J. Wang, ${ }^{1}$ C.Y. Wang ${ }^{1 *}$, Y.T. Feng ${ }^{1}$, C. Tang ${ }^{2}$ \\ 1. Zienkiewicz Centre for Computational Engineering, College of Engineering, Swansea \\ University, Bay Campus, Swansea, SA1 8EN, UK \\ 2. Faulty of Civil Engineering and Mechanics, Jiangsu University, \\ No. 301 Xuefu Road, Zhenjiang, Jiangsu, P.R. China 210013
}

Keywords: Zinc oxide Nanowires, non-linear elasticity, water adsorption, elastic modulus

\begin{abstract}
Surface modification via adsorbates is significant for property prediction in nanostructures where surface effect is dominant. This is especially vital for zinc oxide ( $\mathrm{nnO})$ nanowires (NWs) which has no native passivation layer. As water is an ubiquitous environmental factor and its aggregation on $\mathrm{ZnO}$ surface is favoured, molecular statics (MS) simulations are used to study the deformation of $\mathrm{ZnO}$ with surface water adsorption in the finite strain regime (up to 0.1). Three types of water covered surface structures are considered to examine their effects on the size-dependence of linear $\left(Y_{0}\right)$ and nonlinear $\left(Y_{1}\right)$ elastic moduli. The pathway of adsorption to impact NWs is identified by revealing the radial distribution of $Y_{0}, Y_{1}$ and residual stress for the NWs. The physical origins of the water adsorption effects are further discussed in terms of the layer-wise equilibrium structure and potential energy variation.
\end{abstract}

Corresponding author: chengyuan.wang@swansea.ac.uk 


\section{Introduction}

Wurtzite $\mathrm{ZnO}$ nanowires (NWs) are semiconducting materials with a wide bandgap, piezoelectric character and are biocompatible. The combination of these properties makes them promising for functional components for self-powered nano-electronics ${ }^{1,2}$ and provide an avenue towards prospective innovations in sensing ${ }^{3}$, actuation ${ }^{4}$, piezotronics ${ }^{5,6}$ and biomechanical energy extractors ${ }^{7}$. Particular attention has been paid on characterisation of NW electro-mechanical properties and understanding of the specific surface effects underlining in order to confirm the potential size enhancements and provide guidance on property tailoring in applications. ${ }^{8-10}$

Existing studies of $\mathrm{ZnO} \mathrm{NW}$ mechanical responses have been focused on the measurement of the effective axial Young's modulus and its variation with the characteristic size. ${ }^{11-15}$ This characterisation approach stems from their ability to sustain large and recoverable deformations prior to failure, which is advantageous for $\mathrm{ZnO} \mathrm{NW}$ based piezoelectric and electromechanical devices. It is found in experiment that c-axis ZnO NWs exhibit brittle fracture when subjected to uniaxial loading with failure strain between 0.050.06 and up to a maximum of $0.15 .{ }^{16-19}$ In a more recent investigation by Roy et. al. ${ }^{20}$ fracture strain of 0.06 was also achieved using a two-point bending test. More importantly, no plasticity is evident before failure and a near fully reversable stress-strain response has been confirmed up to uniaxial tensile strain of $0.07 .{ }^{16}$ Therefore, recent first-principle 
characterization of these NWs emphasized capturing finite strain behaviour through third order elastic constants and strain dependent Young's modulus. ${ }^{21-24}$ It was demonstrated that the energy strain relation was well described by a third order polynomial within the strain range of $\pm 0.06-0.08$, showing significant non-linear elasticity where the strain-dependent elastic modulus is required.

It is understood that the elastic behaviour is strongly coupled to surface effects induced by the free surface structural relaxation or reconstruction..$^{25-27}$ As such, modification of the equilibrium structure of pristine surfaces by adsorbates from the surrounding environment can make tangible alterations to elastic properties and desired functions. In this regard, the water-ZnO interface is an ideal system to consider due to the existence of moisture in most operating conditions and the wealth of literature on water adsorption and aggregation behaviour on $\mathrm{ZnO}$ free surfaces. ${ }^{28-32}$ Indeed a previous experiment based on AFM three-point bending has observed water adsorption induced stiffening of $\mathrm{ZnO}$ nanobelts. ${ }^{33} \mathrm{~A}$ remarkable increase of Young's modulus from $40 \mathrm{GPa}$ at $10 \%$ relative humidity to $88 \mathrm{GPa}$ at $80 \%$ relative humidity was measured. The accompanying first-principles adsorption simulation and analytical model attributes the stiffening to the reduction of compressive surface residual stresses brought on by the near complete removal of surface relaxations. ${ }^{33}$ Furthermore, water adsorption impact on ZnO NW piezoelectricity was studied using molecular dynamics and analytical modelling where reduction of the piezo-potential generated is observed for 
higher humidity levels or surface water coverage. A down shift of the conduction band edges is also observed but found to have a trivial influence on the overall piezopotential. ${ }^{34}$ Hence, with substantial demonstrable NW property alternations observed it is inherently valuable to further quantify the adsorption influence on the elastic properties of $\mathrm{ZnO} \mathrm{NWs}$ and attempt to draw a mechanistic understanding.

To the authors' best knowledge, the direct examination of the water adsorption influence has not been conducted for $\mathrm{ZnO}$ NWs elastic response and size dependence trend with in the non-linear regime. As consistent experimental demonstration of non-linear elasticity is not available - due to difficulty over structural control - we first demonstrate that non-linear elasticity can be significant for NW elasticity even for diameters $>15 \mathrm{~nm}$ (the experimentally accessible range ${ }^{8}$ ), which can be characterised by the second order (Young's modulus) and the third order elastic modulus of the NWs. These overall elastic properties are computed for pristine and water-passivated NWs based on the molecular statics method. Then qualification of water adsorption impact on NW elasticity is drawn through the variation of these three parameters and their size dependence trend upon $1 \mathrm{ML}$ water passivation. To gain mechanistic insights, the surface effect is examined through layer-wise elastic property computation and its influence is discussed in terms of the induced axial residual stress and layer equilibrium length change. Finally, by comparing the pristine and water passivated 
surface effect and the differing resultant layer-wise elasticity, the influence of water adsorption modification on overall NW $Y_{0}$ and $Y_{1}$ is explained.

\section{Methodology}

\subsection{Passivated nanowire model}

As an exemplar study of water adsorption effect on $\mathrm{ZnO} \mathrm{NW}$ elasticity the hexagonal [0001] oriented (c-axis) ZnO NWs with $\{01 \overline{1} 0\}$ facets are examined here for diameters of $2.2 \mathrm{~nm}, 5 \mathrm{~nm}, 10 \mathrm{~nm}$ and $15 \mathrm{~nm} \cdot{ }^{35,36}$ Larger sized NWs are not modelled as convergence of elastic property is observed at $15 \mathrm{~nm}$. The pristine c-axis NW structure is detailed in Fig. 1a. This NW orientation is selected as it is one of the most prevalent structures synthesized $9,35,37$ and there also exist a wealth of studies ${ }^{28,29,32,38-42}$ of water adsorption behaviour on the $\{01 \overline{1} 0\} \mathrm{ZnO}$ planes to inform the modelling process. In order to capture the water adsorption influence, 1 monolayer $(1 \mathrm{ML})$ water passivated NWs are examined. Although in general, water films formed on free surfaces can span 1-3 layers ${ }^{43-45}$, for $\mathrm{ZnO}$ surfaces the single layer coverage is prevalently observed in experiments $29,32,38,39$. Hence, the $1 \mathrm{ML}$ structure on $\{01 \overline{1} 0\} \mathrm{ZnO}$ facet is examined in order to capture the modifications to NW non-linear elastic responses. An example of the $1 \mathrm{ML}$-passivated NW structure is shown in Fig. $1 \mathrm{~b}$.

At $1 \mathrm{ML}$, the possible water aggregation structures can be described by its degree of dissociation (i.e. The fraction of dissociated water molecules) and is bounded by the higher 
energy fully molecular (FM) (Fig.1c) and fully dissociative (FD) adsorption structure (Fig.1d) ${ }^{39}$. Here molecular adsorption occurs via bonding of surface $\mathrm{Zn}$ atom, $\mathrm{Zn} \mathrm{n}_{\mathrm{s}}$, and oxygen of the water molecule, $\mathrm{O}_{\mathrm{w}}$ (Fig.1c). For dissociative adsorption, the water molecule breaks into $\mathrm{OH}$ group and $\mathrm{H}$ forming both $\mathrm{Zn}_{\mathrm{s}}-\mathrm{O}_{\mathrm{OH}}$ and $\mathrm{O}_{\mathrm{s}}-\mathrm{H}$ bonds (Fig.1d). For $\mathrm{ZnO}\{01 \overline{1} 0\}$ surfaces ${ }^{31,46}$ computational and experimental study under ultra-high vacuum have shown that, in the coverage regime $\leq 1 \mathrm{ML}$, the half-dissociative (HD) adsorption structure (Fig.1e) with 1:1 ratio of dissociated and molecularly adsorbed water molecule is most energetically favorable. However constant interconversion occurs between these key structures as transition energy between them is small $(0.02 \mathrm{eV}){ }^{31,38}$ and recently $\mathrm{ML}$ fully dissociative coverage has been found under higher relative humidity conditions ${ }^{32}$. Thus in this study, we elect to compute uni-axial mechanical responses of the NWs covered by each of the three $1 \mathrm{ML}$ structure to distinguish their individual impact on elasticity as well as obtain a semi-quantitative gauge on the range of variation for the size effect trend.

The structural models of the 1ML-passivated NWs are constructed by placing water molecules on the surface of a NW with bulk lattice constants. The placement sites are selected based on the energetically favourable adsorption structure on an infinite $\mathrm{ZnO}\{01 \overline{1} 0\}$ plane found by first-principles studies ${ }^{30,31}$. The initial NW structures are then equilibrated using the ReaxFF potential ${ }^{30}$ and the obtained equilibrium water covered $\mathrm{ZnO} N W\{01 \overline{1} 0\}$ facets are shown in Fig.1 c-e for all three adsorption types. The optimized structures are in reasonably 
good agreement with existing first-principles study in terms of the bond lengths and angles between the NW surface and adsorbate atoms. Differences in the periodicity of the equilibrium water monolayer observed is primarily due to the fact that the first-principles studies ${ }^{30,31}$ considered an infinitely large surface while a finite surface with edge/corner effect is studied here (Please refer to Supplementary materials for detailed comparison between the adsorption structures obtained by the current simulation and the first-principles study ${ }^{31}$ ).

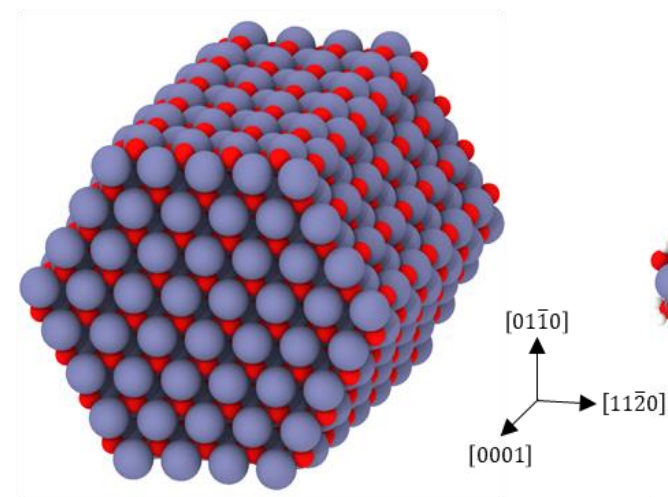

(a)

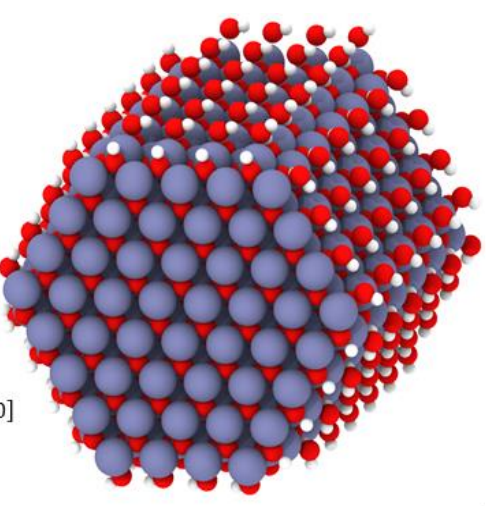

(b)

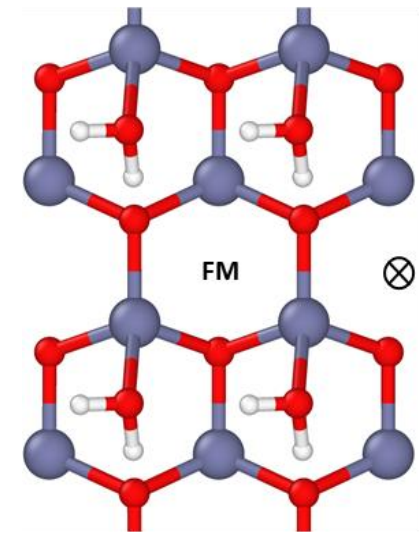

(c)

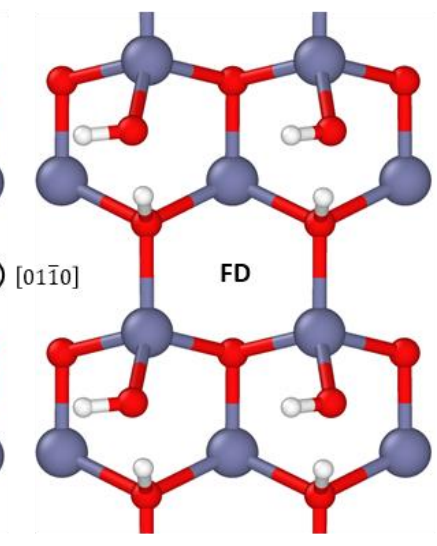

(d)

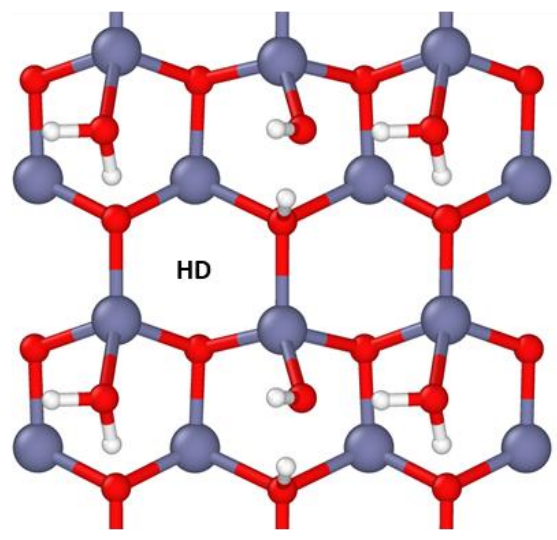

(e)

Figure 1. C-axis ZnO NW structure. a) Pristine NW. b) $1 \mathrm{ML}$ water-passivated NW. The equilibrium FM (fully molecular), FD (fully dissociative) and HD (half-dissociative) water adsorption structures on $\{01 \overline{1} 0\} \mathrm{ZnO} N W$ facets are shown respectively in (c), (d) and (e). The constituent $\mathrm{Zn}$ atoms are coloured grey, $\mathrm{O}$ atoms red and $\mathrm{H}$ atoms white. 


\subsection{Interaction potential}

To describe the atomic interactions in the $\mathrm{ZnO}$-water system, the reactive force field ReaxFF is utilised. First developed for hydrocarbons by Van Duin et. al. ${ }^{47}$ ReaxFF has been successfully extended to ZnO systems and their interaction with water ${ }^{30,48,49}$. The total interaction energy decomposition is described in Eqn. 1.

$$
E_{\text {system }}=E_{\text {bond }}+E_{\text {over }}+E_{\text {under }}+E_{\text {lp }}+E_{\text {val }}+E_{\text {tors }}+E_{v d W a a l s}+E_{\text {Coulomb }}
$$

The first six terms stand, sequentially, for the bond energies, over- and under- coordination penalty energies, lone pair energy, valence energy, and torsion angle energy. These terms describe the bonded interactions and are dependent on the bond-order. The final two terms stand for the Van der Waal (vdW) energy and Coulombic energy, which are screened and shielded to limit strong repulsion at short distances. These non-bonded interactions are calculated based on a geometrically dependent charge distribution obtained through the charge equilibration method. The above features of ReaxFF enable the depiction of charge transfer and hence bond breaking and formation, which is critical to the appropriate modelling of interfacial behaviour for complex systems. The ReaxFF ZnO potential used in this study is the form adopted to the LAMMPS molecular dynamics software suite ${ }^{50}$ with parameters from the reference ${ }^{30}$. The potential well reproduces $\mathrm{ZnO}$ structural, elastic, vibrational and thermodynamic properties in the bulk and on a range of $\mathrm{ZnO}$ free surfaces compared to experimental and first-principles data ${ }^{30}$. The water adsorption structure 
predicted for various $\mathrm{ZnO}$ free surfaces coheres well with first-principles predictions. Especially, the energetically favourable half-dissociative equilibrium adsorption structure is successfully obtained at $300 \mathrm{~K}$ for $\{01 \overline{1} 0\}$ surfaces $^{30,49}$, which is key reason for its application in the studies of the wetting mechanics on $\mathrm{ZnO}$ surfaces and the humidity influence on the caxis ZnO NW piezo-potential generation. ${ }^{34,51}$ Most importantly, the structural relaxation - a vital part of the surface effect - on the pristine free surface as well as its change due to water adsorption is reproduced.

\subsection{Elasticity characterization}

The LAMMPS software suite is utilised to conduct simulated uniaxial deformation test at OK for the considered NWs utilizing the molecular statics (MS) method. ${ }^{50}$ In all cases, periodic boundary condition is enforced in the axial direction to remove the end effect. The initial structure associated with local energy minimum is found through energy minimization via the conjugate gradient method. Homogeneous axial strain, $\varepsilon$, is then applied in increments of \pm 0.0025 up to \pm 0.08 . The equilibrated structure at each strain state is again found through minimization of the energy via the conjugate gradient method and the total energy of the NW and the individual hexagonal concentric layers are outputted.

The total energy, $\mathrm{E}_{\text {system, }}$ at equilibrium is computed by the sum of the energies of atoms constituting the considered NW or layers. Note that the total energy is the sum of the strain 
energy and the internal energy at zero strain, and is normalized by the number of $\mathrm{Zn}-\mathrm{O}$ dimers in the NW to evaluate the total energy per ZnO dimer, $E_{a}$. The dimer normalized energy, $E_{a}$, is used instead of the energy per unit volume as the enveloping volume of an NW is not uniquely defined and especially so, upon inclusion of a water adlayer. In the case of water passivated NWs (and associated surface layer), $E_{a}$ is computed by including the total energy of the water adlayer (normalized by number of water molecules) in order to account for all strain energy stored. To compute the constitutive relation between the strain energy and the
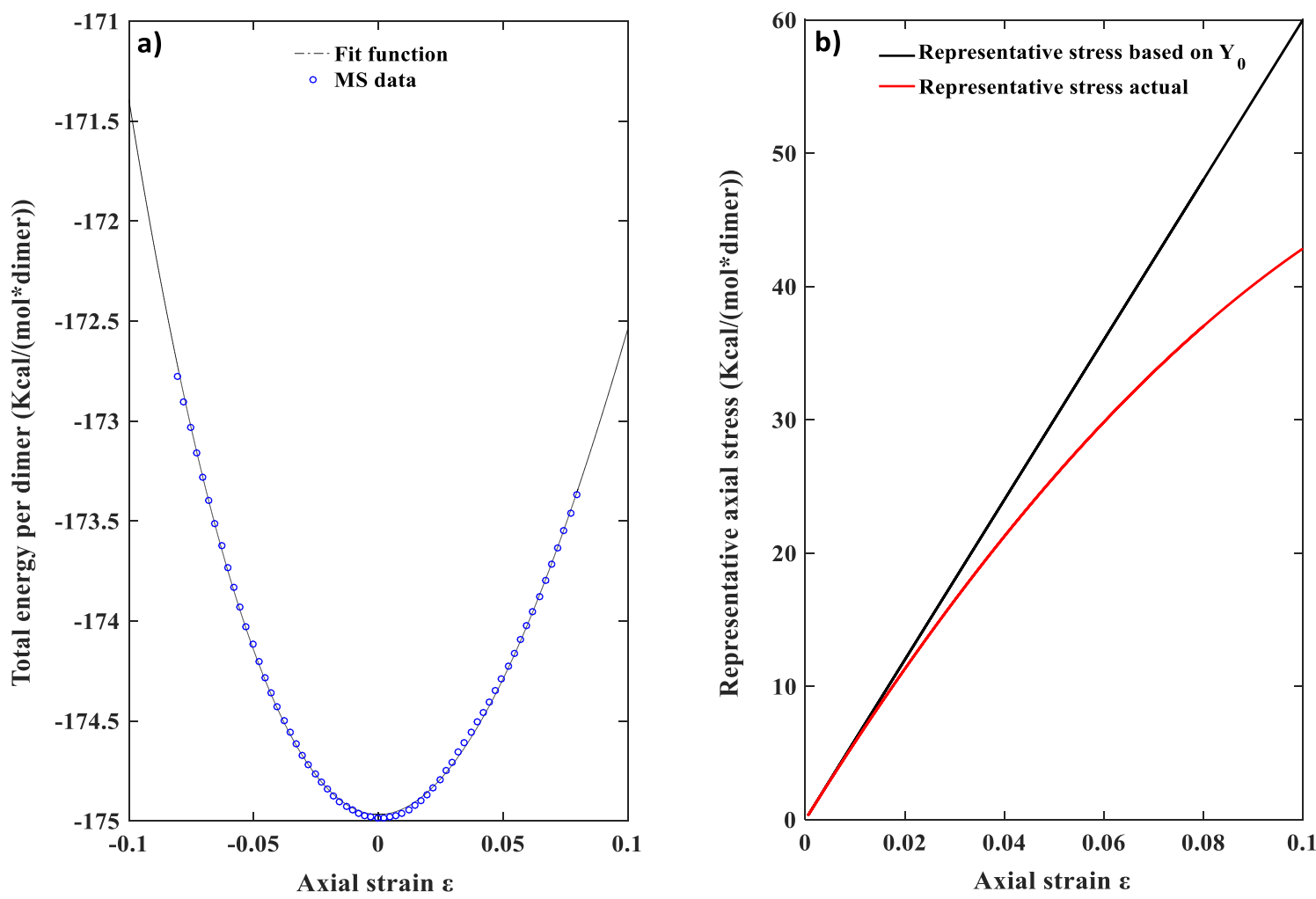

Figure 2. a) Total energy per dimer, $E_{a}$, to axial strain, $\varepsilon$, relationship for Pristine $5 \mathrm{~nm}$ NW. The MS data points are shown in blue $(-0.08$ to 0.08$)$ and the strain function constructed by cubic polynomial fitting is shown by the dot-dash line (-0.1 to 0.1$)$. b) Representative axial stress variation with axial strain respectively corresponding to the actual (red) and linear elastic portion (i.e. $Y_{0} \cdot \varepsilon$; black) of the relation for $5 \mathrm{~nm}$ pristine NW. The nonlinear elastic contribution is the difference between the two relations. 
axial strain, the $E_{a}-\varepsilon$ relationship is plotted in the strain range of $[-0.08,0.08]^{22}$ as shown in Fig. $2 \mathrm{a}$ by the blue data points (for $5 \mathrm{~nm}$ NW under $1 \mathrm{ML}$-FM passivation). Then least squares regression using cubic polynomial (Eq. 2) is used to obtain $E_{a}(\varepsilon)$ (dashed line in Fig. 2a).

$$
E_{a}(\varepsilon)=E_{a}(0)+\sigma_{0} \cdot \varepsilon+\frac{Y_{o}}{2} \cdot \varepsilon^{2}+\frac{Y_{1}}{6} \cdot \varepsilon^{3}
$$

The non-linear elasticity of the NW is characterized by the axial strain derivatives of $E_{a}(\varepsilon)$ up to the third order where $\sigma_{0}=\left.\frac{\partial E_{a}}{\partial \varepsilon}\right|_{\varepsilon=0}$ represents the residual stress, $Y_{0}=\left.\frac{\partial^{2} E_{a}}{\partial \varepsilon^{2}}\right|_{\varepsilon=0}$ the effective Young's modulus, $Y_{1}=\left.\frac{\partial^{3} E_{a}}{\partial \varepsilon^{3}}\right|_{\varepsilon=0}$ the effective nonlinear axial modulus. This leads to a strain-dependent tangent modulus $Y_{0}+Y_{1} \varepsilon$. Our results show that the overall residual stress is negligibly small and thus will not be considered in the analysis of overall NW elasticity.

\section{Results and discussions}

In this section, the characterization of the tensile response of the $\mathrm{ZnO}$ NWs under finite strain is conducted based on the above MS technique. The significance of the nonlinear modulus, the influence of the $1 \mathrm{ML}$ water passivation on the nonlinear elastic responses and the physical mechanisms underlying the observations will be examined.

\subsection{Nonlinear elasticity in NWs}

The non-linearity in the elastic response of NW is discussed by first comparing the actual nonlinear stress-strain relation to the associated linear component. Specifically, for the $5 \mathrm{~nm}$ pristine NW, the actual elastic stress-strain relation (i.e. $\sigma_{a c t}=\frac{\partial \overline{\mathrm{E}}_{a}(\varepsilon)}{\partial \varepsilon}$ and in red) and its linear elastic component (i.e. $\sigma_{l}=Y_{o} \varepsilon$ and in black) are plotted in Fig. $2 \mathrm{~b}$. It is seen from Fig.2b that 
the total strain energy (i.e. $E_{S, a c t}=\sigma_{0} \cdot \varepsilon+\frac{Y_{O}}{2} \cdot \varepsilon^{2}+\frac{Y_{1}}{6} \cdot \varepsilon^{3}$ and the area below the red line) is lower than the linear strain energy portion (i.e. $E_{S, Y_{O}}=\frac{Y_{O}}{2} \cdot \varepsilon^{2}$ and the area under the black line) indicating negative $Y_{1}$ or the strain softening of the NWs.

To quantify the observed non-linearity, the critical strain $\varepsilon_{c r}$ is defined as the axial strain at which $R_{S, Y_{1}}=10 \%$. Here $R_{S, Y_{1}}=-\frac{Y_{1}}{3 Y_{0}} \varepsilon$ is the strain energy ratio between the strain energy from $Y_{1}$ (i.e., $-\frac{Y_{1} \varepsilon^{3}}{6}$ ) and the (linear) strain energy contribution from $Y_{0}$ (i.e., $\frac{Y_{O}}{2} \cdot \varepsilon^{2}$ ). As $R_{S, Y_{1}}$ increases with $\varepsilon$ at a constant rate $\frac{Y_{1}}{Y_{0}}$ the critical strain $\varepsilon_{c r}$ separates the two strain ranges, (1) $\varepsilon \leq \varepsilon_{c r}$ associated with $R_{S, Y_{1}} \leq 10 \%$, i.e., primarily linear elastic response and (2) $\varepsilon>\varepsilon_{c r}$ corresponds to $R_{S, Y_{1}}>10 \%$, i.e., nonlinear elastic behavior of the NWs. Since the

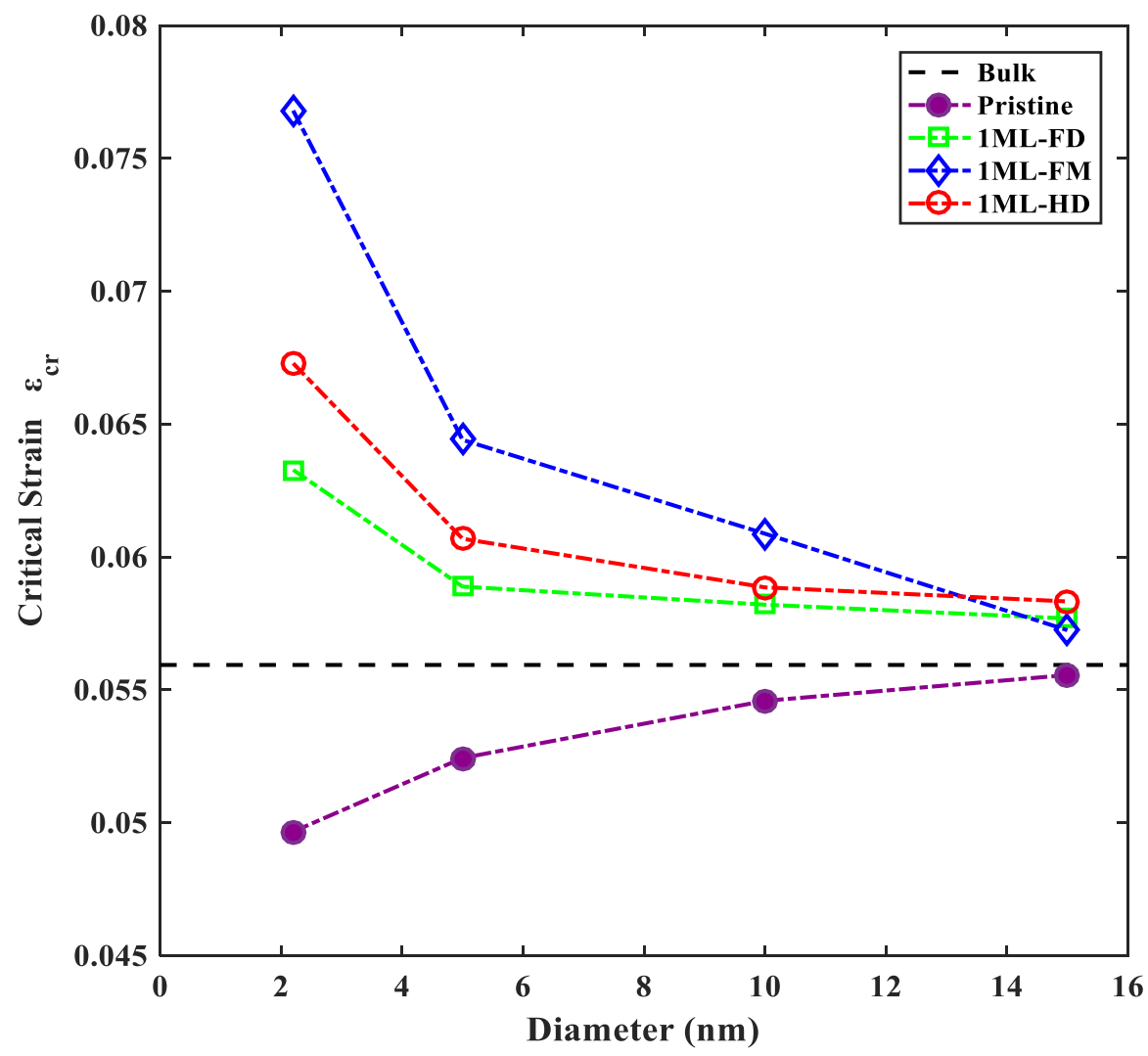

Figure 3. Critical strain at $R_{s, Y_{1}}=10 \%$ calculated for pristine (purple), $1 \mathrm{ML}-\mathrm{FM}$ (blue), 1ML-HD (red) and 1ML-FD (green) passivated NWs of diameters 2.2, 5, 10 and 15nm. The bulk $\mathrm{ZnO}$ value of 0.056 is indicated by the horizontal dashed line. 
current and existing studies do not find plasticity prior to failure for c-axis $\mathrm{ZnO} \mathrm{NWs}{ }^{15,16}, R_{S, Y_{1}}$ is evaluated for all NWs considered up to strain values of 0.1 , which is the mean of the failure strain range $[0.05,0.15]$ reported in literature. ${ }^{17-19}$ The critical strain $\varepsilon_{c r}$ to diameter relation for all NWs are presented in Fig. 3. For pristine NWs (purple circles) $\varepsilon_{c r}$ increases as diameter enlarges and nearly reaches the asymptotic value of 0.056 at $15 \mathrm{~nm}$. The convergence agrees with the linear elastic regime $[0,0.062]$ measured for 20 to $80 \mathrm{~nm}$ c-axis ZnO NWs through Insitu AFM tension by Xu et. al. ${ }^{16}$. Considering the asymptotic $\varepsilon_{c r}$ value of 0.056 and reported ultimate NW failure strains up to 0.15 in experiment and 0.2 in simulations ${ }^{24,52}$, the non-linear component in the c-axis NW elastic response can be substantial not only in the small diameter range considered by computation studies but also in experimentally accessible NWs with diameters greater than $20 \mathrm{~nm}$.

Albeit, it should be pointed out that the observed non-linear elastic response is attributable to the single crystalline structure of the small-size ZnO NWs studied in the present and previous studies. Thus nonlinear elasticity in a wider strain range is a physical characteristic which distinguishes them from larger ZnO NWs with higher density of defects.

\subsection{Water-passivation effect on the linear-elastic modulus}

The linear elastic moduli for water passivated NWs and their pristine counterparts are presented in Fig. 4 for all considered diameters. In contrast to pristine NWs with $Y_{0}$ increasing with rising diameter, under $1 \mathrm{ML}$ water passivation of all types the overall $Y_{0}$ size-effect trend is inverted, where $Y_{0}$ decreases with rising diameter and approaches the bulk value when the diameter is sufficiently large. 
The sensitivity of $Y_{0}$ to the diameter change decreases due to the water adsorption. At the same NW diameter, 1ML-FM and 1ML-FD passivated, respectively, yield higher $Y_{0}$ values while the $1 \mathrm{ML}-\mathrm{HD}$ structure associated value is lower by $>1.5 \%$. Examining the $2.2 \mathrm{~nm} \mathrm{NWs}$, the relative increase against the pristine $Y_{0}$ value for $1 \mathrm{ML}-\mathrm{HD}, 1 \mathrm{ML}-\mathrm{FD}$ and $1 \mathrm{ML}-\mathrm{FM}$ is $20.6 \%$, $22.1 \%$ and $22.4 \%$, respectively. Evidently, $1 \mathrm{ML}$ water adsorption can make the NWs substantially stiffer. This relative increase of $Y_{o}$ in Fig. 4 declines upon the increase of the NW diameter and becomes $\sim 3 \%$ at $15 \mathrm{~nm}$.

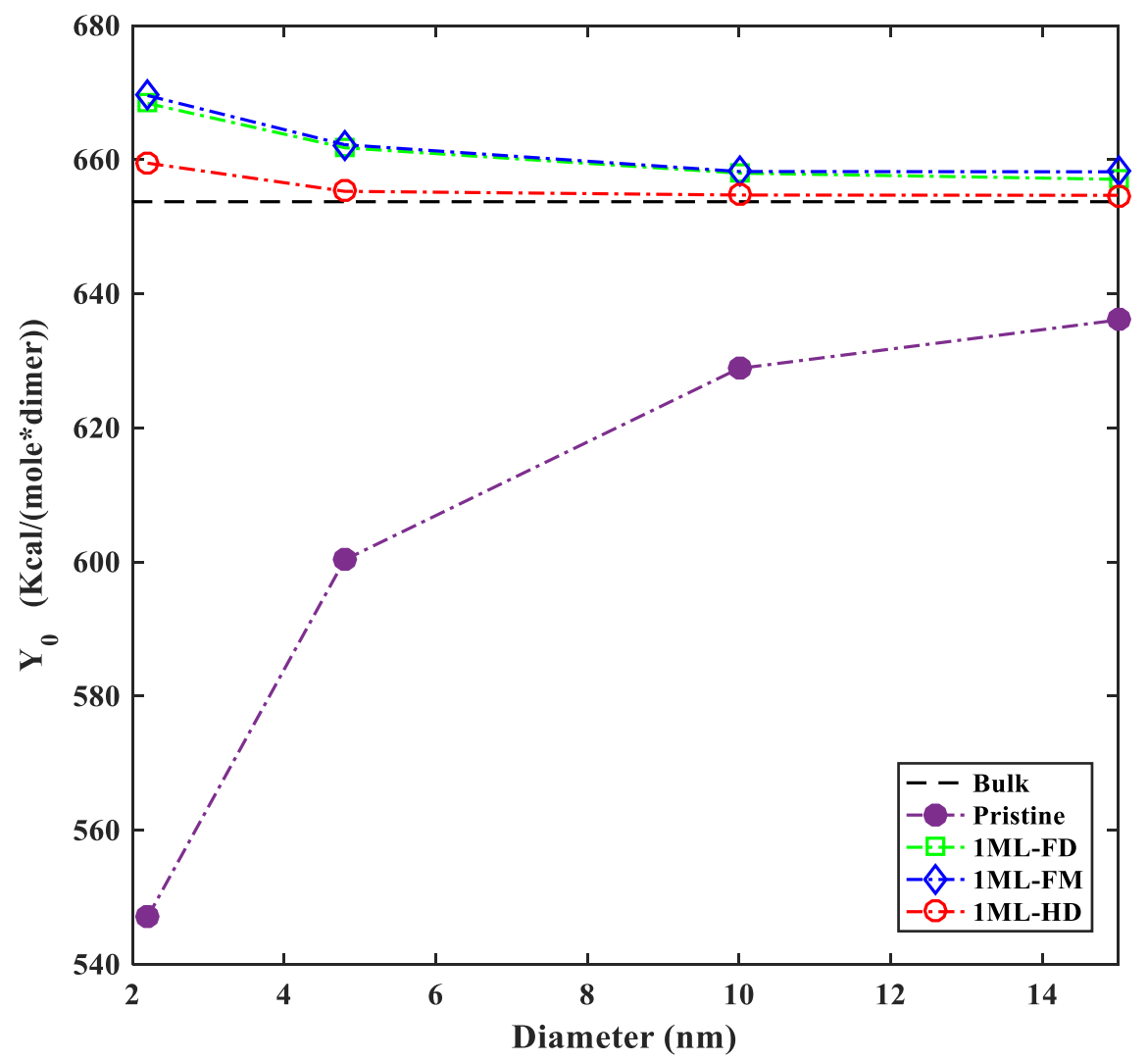

Figure 4. $\mathrm{Y}_{0}$ variation with diameter of c-axis NWs, pristine and under $1 \mathrm{ML}$ water passivation, computed using molecular statics energy data for diameters of 2.2, 5, 10 and $15 \mathrm{~nm}$. 
For pristine NWs, it is understood that the surface effect and resultant size-dependent $Y_{0}$ originates from the relaxation of the surface atomic layer due to coordination loss $^{25,26,33,53,54}$. Water adsorption thus chiefly influences the size-dependence of $Y_{o}$ by modifying the surface relaxation and in turn the interior equilibrium structure. To shed light

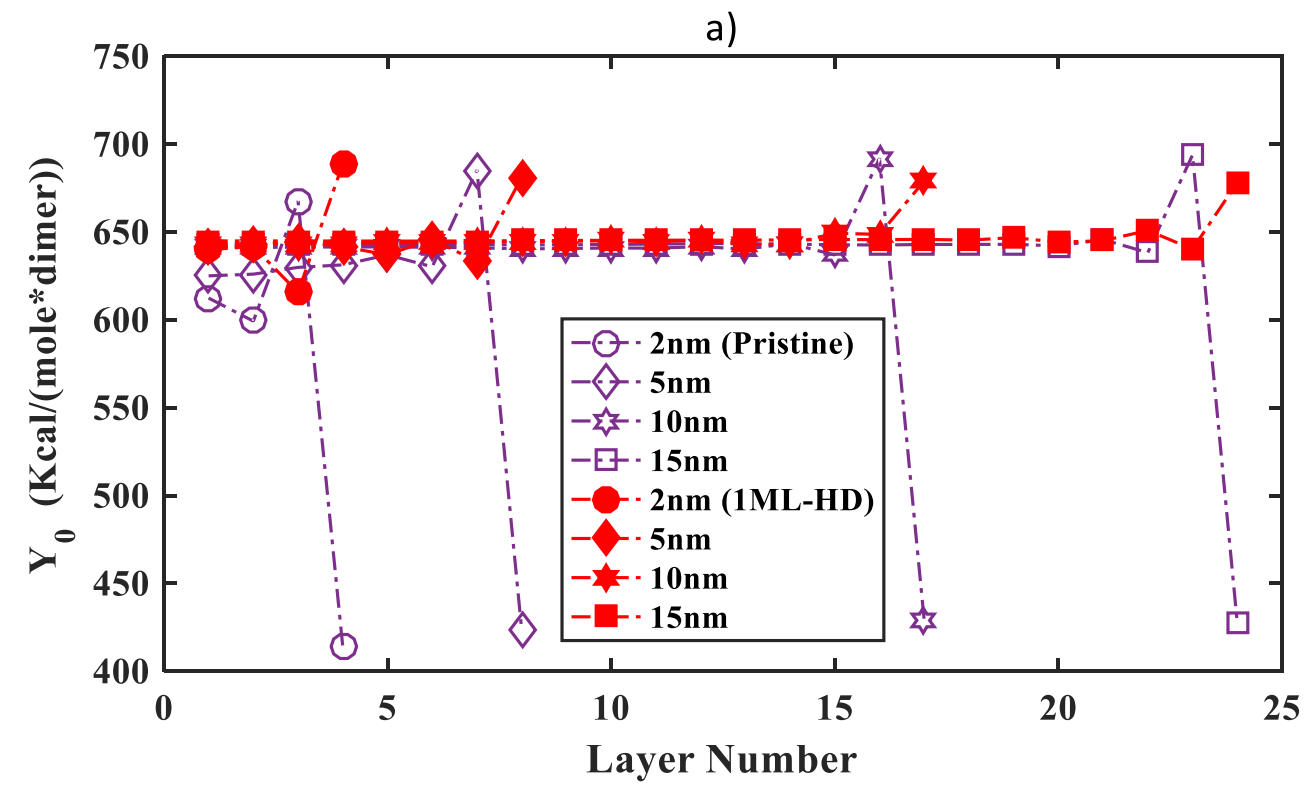

b)

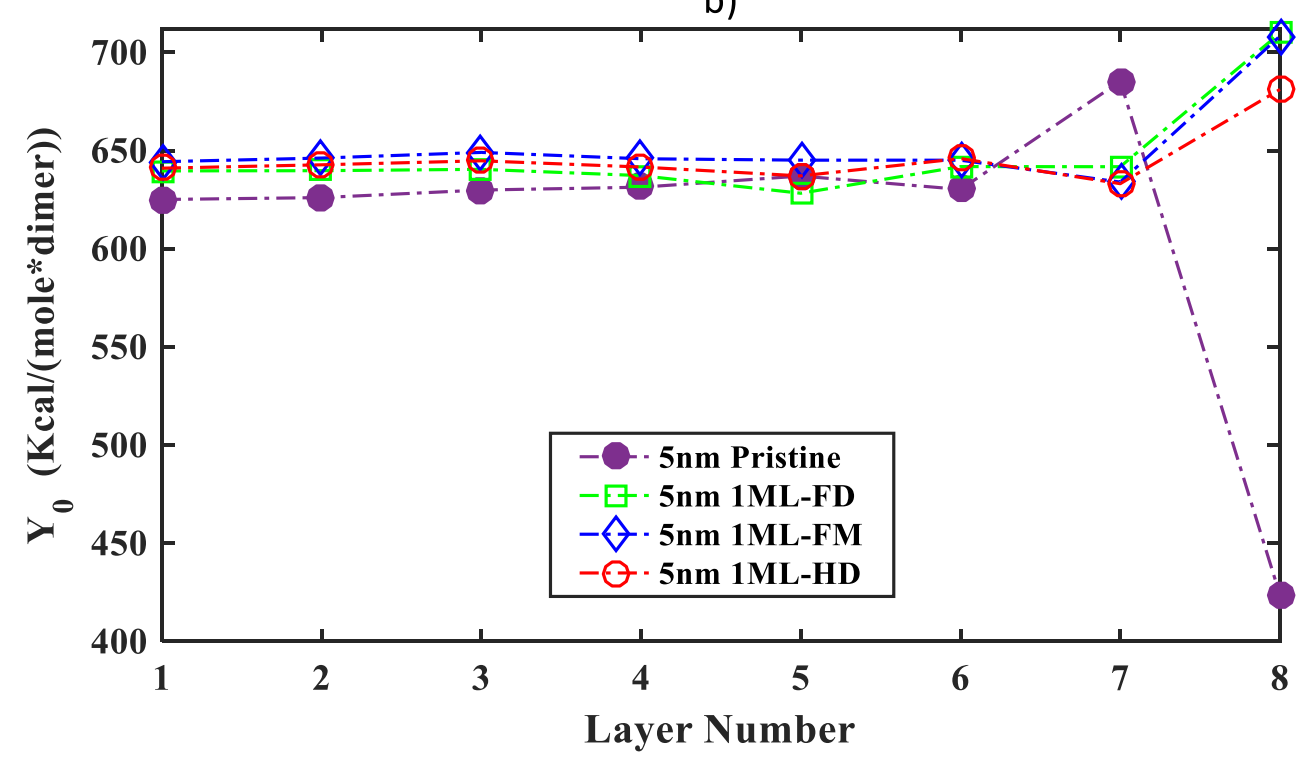

Figure 5. (a) Layer-wise distribution of $Y_{0}$ for pristine and $1 \mathrm{ML}-\mathrm{HD}$ passivated NWs of diameters 2.2, 5, 10 and $15 \mathrm{~nm}$. b) Layer-wise distribution $Y_{0}$ of for $5 \mathrm{~nm} \mathrm{NW}$ with pristine, $1 M L-F D, 1 M L-F M$ and $1 M-H D$ surface. 
on the mechanisms behind water adsorption effects, we calculate the radial distributions of $Y_{o}$ (i.e. $Y_{o}$ of each concentric hexagonal $\mathrm{ZnO}$ layer). The results for pristine and $1 \mathrm{ML}-\mathrm{HD}$ passivated NWs are shown in Fig. 5a for diameters $2.2 \mathrm{~nm}, 5 \mathrm{~nm}, 10 \mathrm{~nm}$ and $15 \mathrm{~nm}$, respectively. For water passivated NWs the highest $Y_{o}\left(\sim 680 \mathrm{Kcal} /\left(\mathrm{mol}^{*}\right.\right.$ dimer $\left.)\right)$ is obtained at the outmost (surface) layer and $Y_{o}$ of the inner layers are approximately constant with values slightly lower than the $653 \mathrm{Kcal} /\left(\mathrm{mol} *\right.$ dimer) bulk value. In contrast, for pristine NWs the lowest $Y_{o}$ is found at the surface layer and $Y_{o}$ of the inner layers remains are also near constancy with values between $600-650 \mathrm{Kcal} /\left(\mathrm{mol}^{*}\right.$ dimer$)$.

Based on the core-shell model ${ }^{55}$ it is evident from Fig. 5a the increase of the overall $Y_{O}$ with diameter increase obtained for pristine NWs (Fig. 4) is a result of the low surface $Y_{o}$. The opposite size-dependence of $Y_{o}$ observed for water passivated NWs thus originates from the high surface $Y_{o}$ due to adsorption. In addition, the difference in $Y_{o}$ between the surface and the inner layers is $\sim 6.8 \%$ for water passivated NWs, which is much smaller than the $\sim 48 \%$ obtained for the pristine NWs and thus leads to the less pronounced size-dependence of $Y_{O}$ (Fig. 4). A similar water adsorption effect on the surface $Y_{o}$ is obtained for all three water adsorption modes as exemplified in Fig. $5 \mathrm{~b}$ for the NWs of diameter $5 \mathrm{~nm}$.

To further reveal the mechanism behind the above surface effect and water adsorption influence we calculate the radial distribution of the representative residual axial stress $\sigma_{o}$. The results are shown in Fig. 6a for pristine and 1ML-HD covered NWs of different diameters and Fig. $6 \mathrm{~b}$ for pristine and three different water passivated NWs of the same diameter $5 \mathrm{~nm}$. Here we note that the elastic parameters of each concentric layer is calculated at the equilibrium state of the entire NW cohering with $\sigma_{0}=\left.\frac{\partial E_{a}}{\partial \varepsilon}\right|_{\varepsilon=0}$ and $Y_{0}=\left.\frac{\partial^{2} E_{a}}{\partial \varepsilon^{2}}\right|_{\varepsilon=0}(\varepsilon$ is 
the axial strain of the NW). In this case, the surface layer of the pristine NWs is under compression while the inner layers are in tension (Fig. 6). Therefore, the surface layer compressed in the NW should elongate axially to reach its natural equilibrium state when the

a)

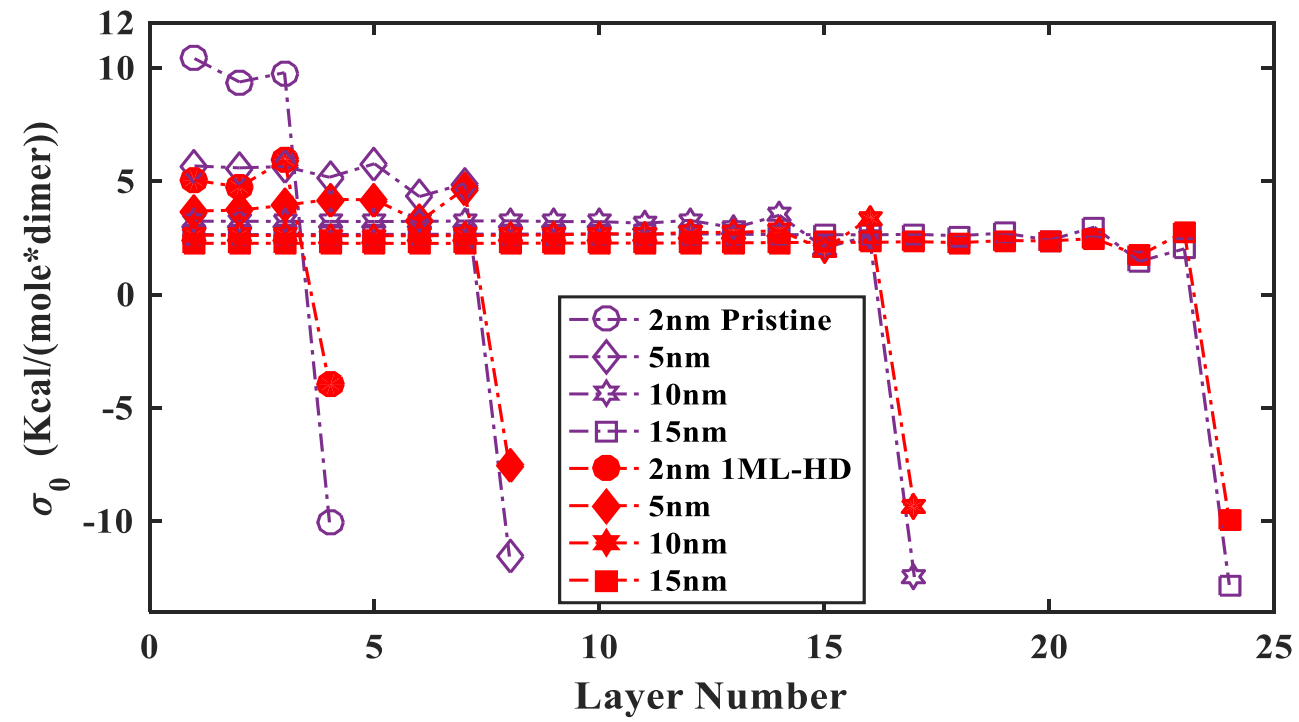

b)

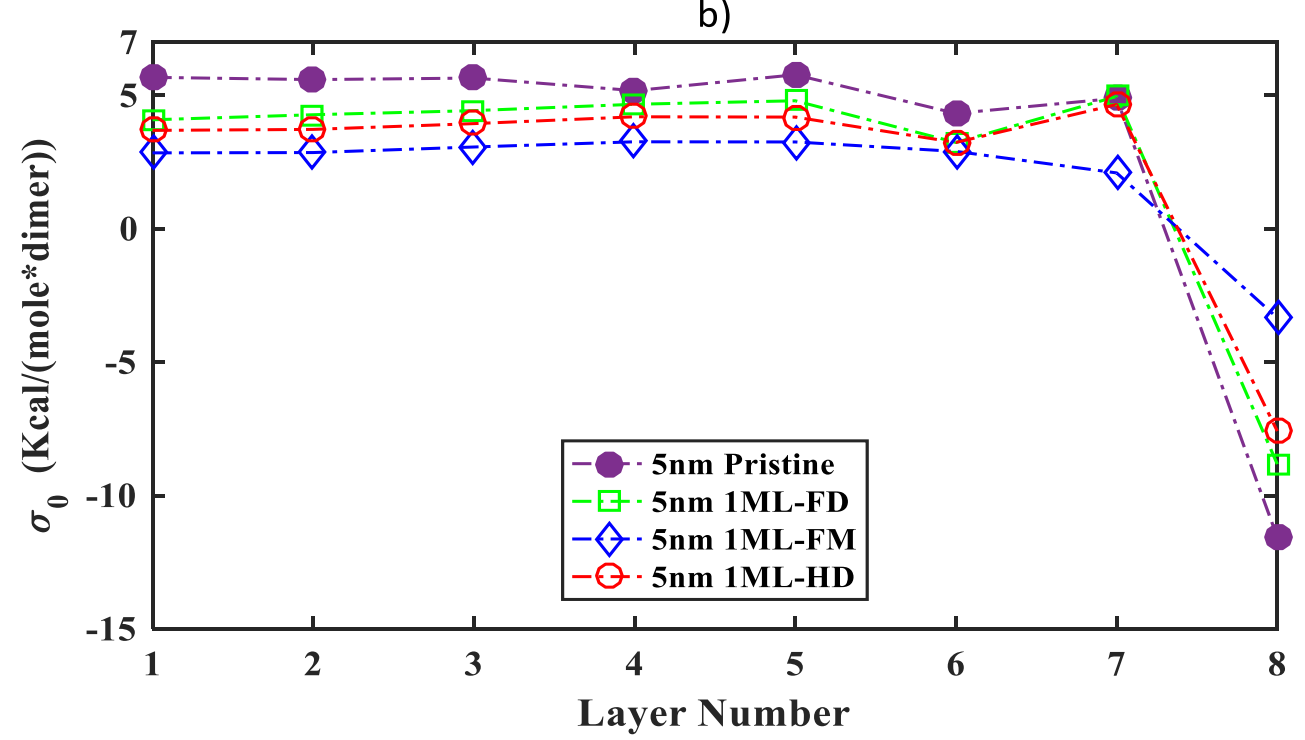

Figure 6. (a) Layer-wise distribution of $\sigma_{0}$ for pristine and 1ML-HD passivated NWs of diameters $2.2,5,10$ and $15 \mathrm{~nm}$. b) Layer-wise distribution $\sigma_{0}$ of for $5 \mathrm{~nm} \mathrm{NW}$ with pristine, 1ML-FD, 1ML-FM and 1M-HD surface. 
residual compressive strain is fully removed. The inner layers, on the other hand, should shrink if the residual tensile strain is fully eliminated and approach their natural equilibrium state. In other words, at the natural equilibrium state without residual stress, the surface layer holds a greater c-axis lattice constant than their counterpart of the inner layers. It is understood that $Y_{0}=\left.\frac{\partial^{2} E_{a}}{\partial \varepsilon^{2}}\right|_{\varepsilon=0}$ calculated for individual layers has two components, i.e., $\frac{\partial^{2} E_{a}}{\partial \varepsilon^{2}}$ obtained at their natural equilibrium state without residual strain and the component arising from the strain softening due to the residual strain. Further analysis show that the $Y_{0}$ of individual layers is predominantly determined by the first component obtained when the residual strain is zero. For the surface layer the lower Young's modulus $Y_{0}$ is thus a result of the lower value of its first component obtained partially due to the larger c-axis lattice constant at the natural equilibrium state.

When $1 \mathrm{ML}-\mathrm{HD}$ is added to the NWs (Fig. 6a), the compressive stress on the surface layer is reduced from the pristine value by $22 \%-60 \%$ and reduction of the tensile stress on the inner layers follows. Similar behavior is also observed for other types of adsorption in Fig. $6 \mathrm{~b}$. This tangible reduction suggests that the adsorption of water reduces the equilibrium axial length or c-axis constant of the surface layer relative to those of the pristine surface and based on the theory proposed above, improves $Y_{o}$ of the surface layer. However, the surface layer of water covered NWs is still subject to compression and thus, its c-axis constant at natural equilibrium should still be longer than that of the inner layers. Thus, the observation that $Y_{o}$ of the surface layer covered by water being greater than that of inner layers (Fig. 5) cannot be fully explained by the decreased c-axis constant. Other factors, e.g., the additional energy 
stored in the water molecules and the restoration of surface $\mathrm{Zn}$ or $\mathrm{O}$ to a fully coordinated state, should have a significant role to play in determining the surface $Y_{o}$. This issue deserves to be further examined in detail.

\subsection{Water-passivation effect on the nonlinearity of NW elasticity}

In this section, we shall discuss the influence of the water adsorption on the nonlinear elastic modulus $Y_{1}$, which measures the magnitude of nonlinearity or the strain softening effect. In Fig. 7, $Y_{1}$ is calculated for the pristine and three types of water passivated NWs of all diameters considered. It is clear that the water passivated NWs retain a similar size-

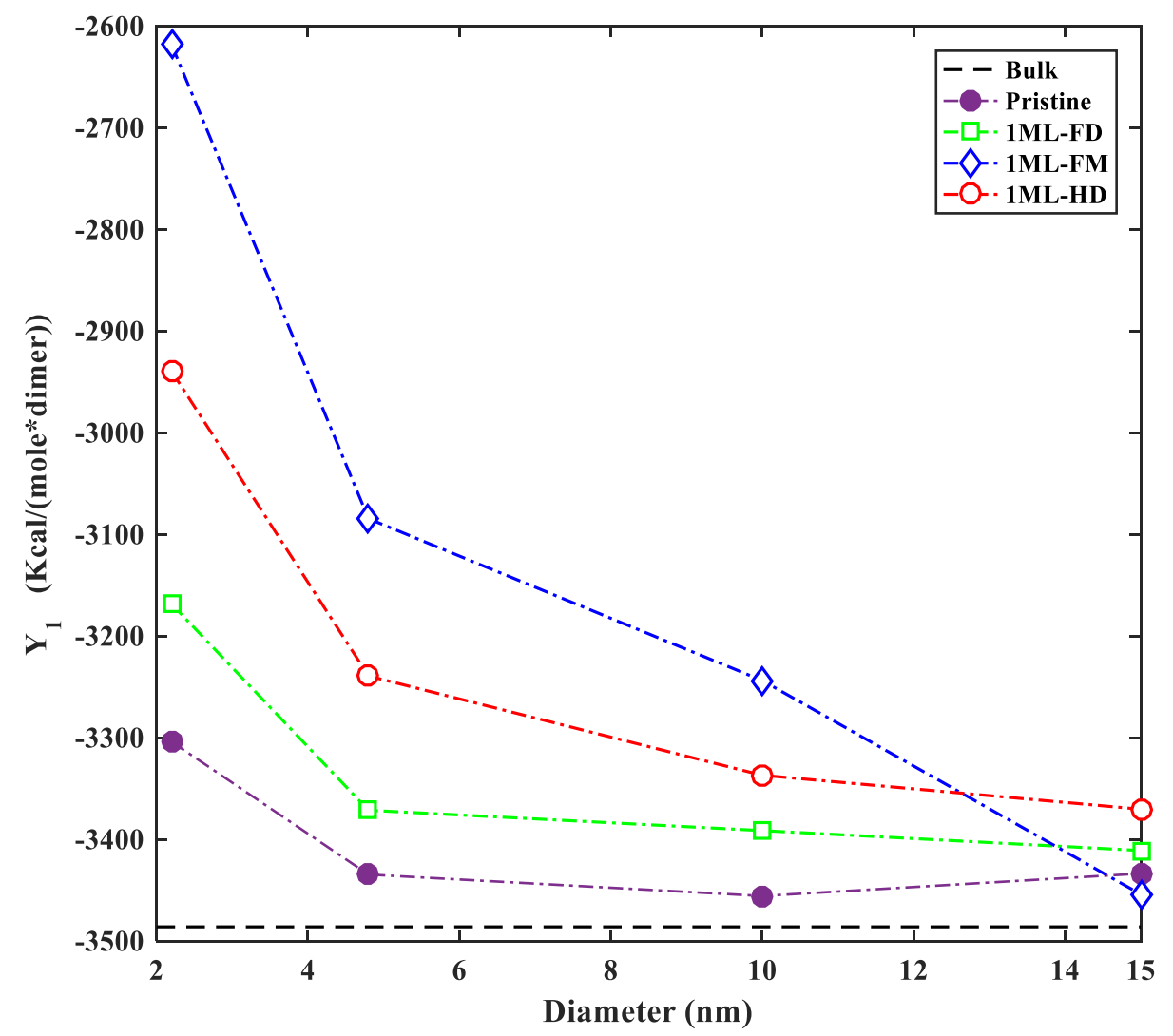

Figure 7. $Y_{1}$ variation with diameter of $c$-axis $N W s$, pristine and under $1 M L$ water passivation, computed using molecular statics energy data for diameters of 2.2, 5, 10 and $15 \mathrm{~nm}$. 
dependence trend for $Y_{1}$, where $Y_{1}$ decreases (i.e., its absolute value increases) with diameter increase. With respect to the adsorption structure, the rate of $Y_{1}$ variation with diameter and the value of $Y_{1}$ increases sequentially for $1 \mathrm{ML}-\mathrm{FD}, 1 \mathrm{ML}-\mathrm{HD}$ and $1 \mathrm{ML}-\mathrm{FM}$. At $2.2 \mathrm{~nm}$ diameter, $1 \mathrm{ML}-\mathrm{FD}$ yields $Y_{1}$ increasing against the pristine value of $4 \%$ followed by $11 \%$ for $1 \mathrm{ML}-\mathrm{HD}$, and lastly $21 \%$ for $1 \mathrm{ML}-\mathrm{FM}$. This relative increase of $Y_{1}$ is reduced to $<1.8 \%$ at $10 \mathrm{~nm}$ and $15 \mathrm{~nm}$ for all three adsorption types suggesting a surface dominant water adsorption modification. Clearly, irrespective of the adsorption type and NW diameter, greater $Y_{0}$ (Fig. 4) and $Y_{1}$ (Fig. 7) or larger effective tangent modulus $Y_{0}+Y_{1} \varepsilon$ are obtained for $1 \mathrm{ML}$ passivated NWs compared to their pristine counterparts. Thus, water adsorption reduces the magnitude of nonlinear strain softening (or water passivated NWs become stiffer) and enhances the sensitive of $Y_{1}$ to diameter change.

The adsorption effect on the extent of nonlinearity can then be examined through $\varepsilon_{c r}$ variation with respect to the pristine NW. Herein, $\varepsilon_{c r}$ variation with diameter for $1 \mathrm{ML}-\mathrm{HD}$, 1ML-FD and 1ML-FM passivated NWs is presented in Fig. 3 in comparison with pristine NWs. As shown in Sec. 3.1 a smaller $\varepsilon_{c r}$ reflects a greater strain range where substantial nonlinear elasticity occurs $\left(R_{S, Y_{1}}>10 \%\right)$. Using the pristine size effect trend as reference, upon $1 \mathrm{ML}$ water passivation this trend is reversed where $\varepsilon_{c r}$ of water-passivated NWs becomes smaller at larger sizes and gradually declines to the bulk value at diameter of $15 \mathrm{~nm}$. For a given diameter $\varepsilon_{c r}$ of water passivated NWs is enhanced relative to the pristine value. Thus, the strain range where substantial nonlinear elasticity occurs is reduced by introduction of surface water adlayer and as diameter decreases. In correspondence with the change in $Y_{1}$, the $\varepsilon_{c r}$ change due to water passivation is very large (maximum of 55\%) at the smallest NW diameter considered but declines to 0.05 when NW diameter approaches $15 \mathrm{~nm}$ (Fig. 3). In addition, the influence of water adsorption becomes greater as 1ML-FD, 1ML-HD and 1ML-FM 
water adsorption are considered in sequence, suggesting a $\varepsilon_{c r}$ dependence on the degree of water dissociation similarly observed in $Y_{1}$.

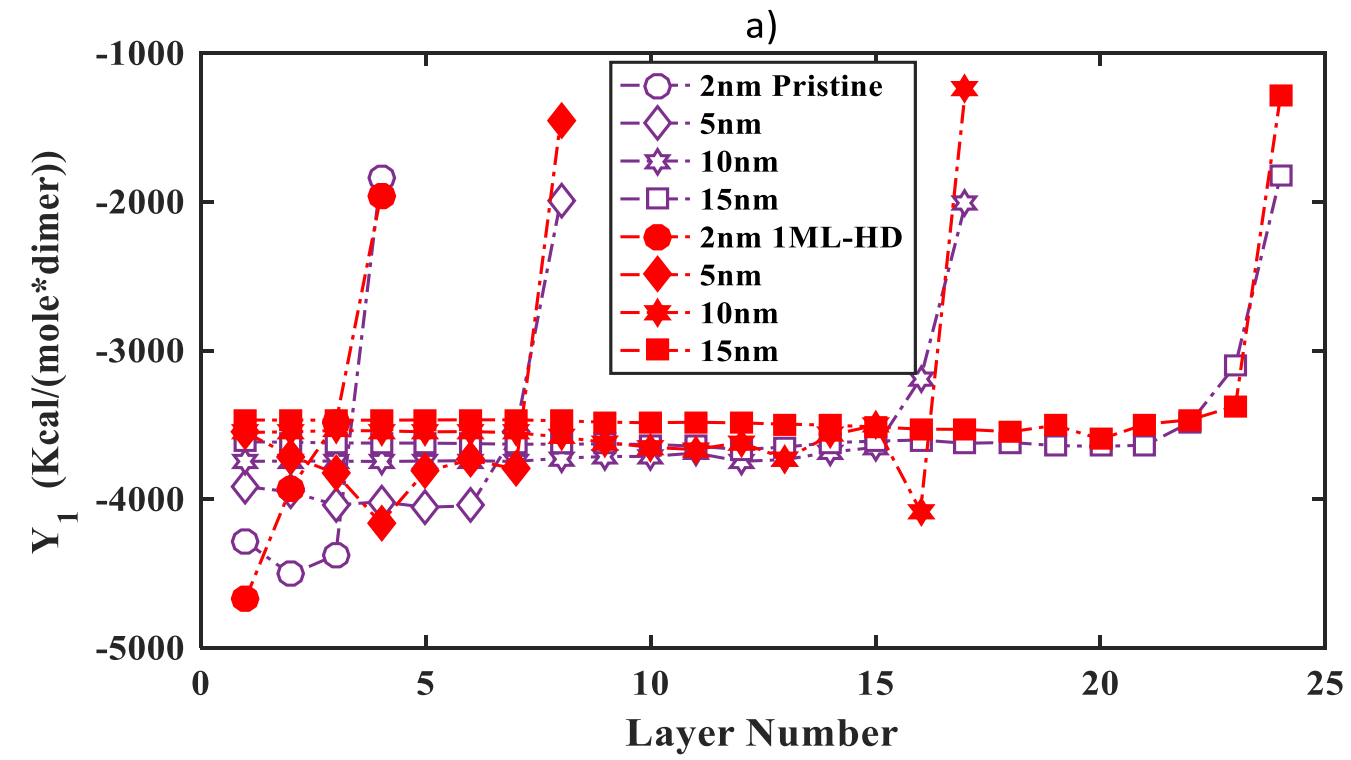

b)

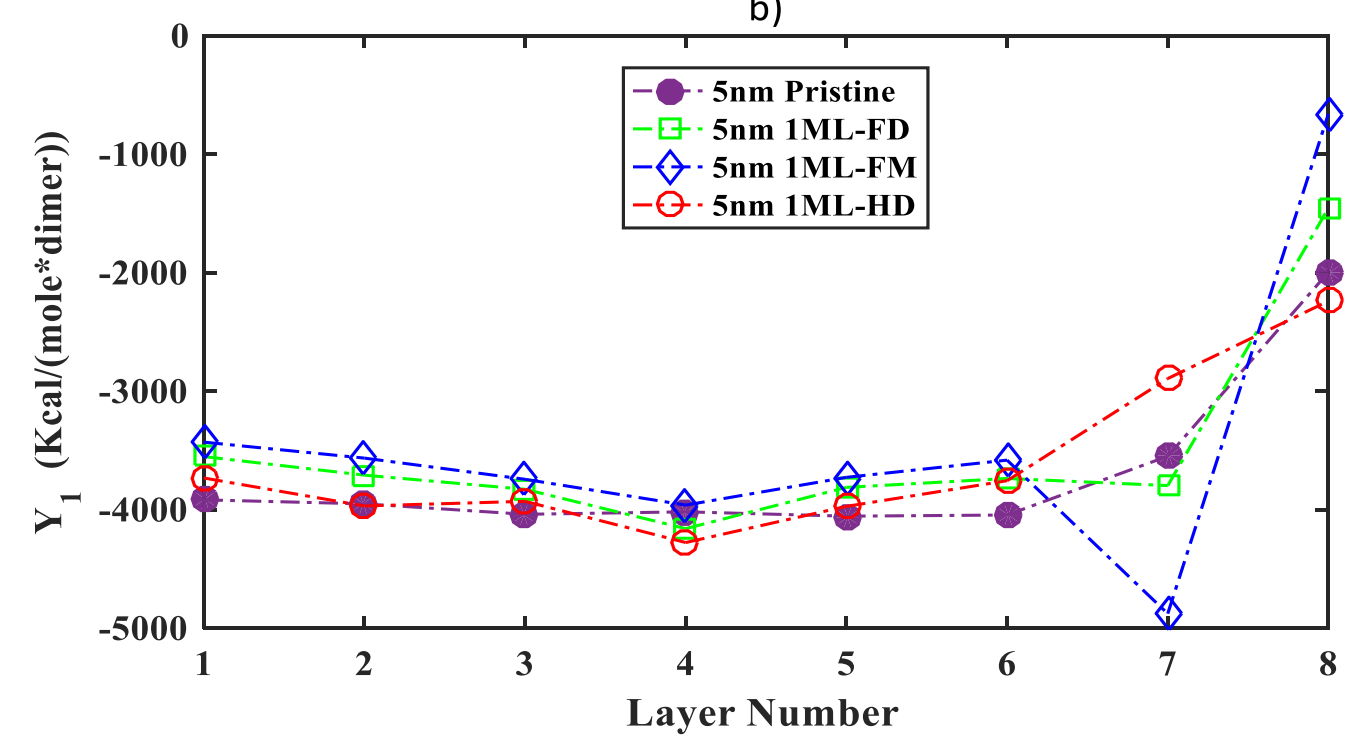

Figure 8. (a) Layer-wise distribution of $Y_{1}$ for pristine and $1 \mathrm{ML}-\mathrm{HD}$ covered NWs of diameters $2.2,5,10$ and $15 \mathrm{~nm}$. b) Layer-wise distribution $Y_{1}$ of for $5 \mathrm{~nm} \mathrm{NW}$ with pristine, 1ML-FD, $1 \mathrm{ML}-\mathrm{FM}$ and $1 \mathrm{M}-\mathrm{HD}$ surface.

To understand the above results, we computed the radial distribution of $Y_{1}$ of the NWs.

The results for pristine and $1 \mathrm{ML}-\mathrm{HD}$ passivated NWs of all considered diameters are shown in 
Fig. 8a and those calculated for 1ML-FM, 1ML-FD and 1ML-HD passivated NWs of diameter $5 \mathrm{~nm}$ are compared in Fig. 8b. Evident in Fig. 8a, $Y_{1}$ of the surface atom layer is larger (or $\left|Y_{1}\right|$ is smaller) than that of all the inner layers. This indicates that the size-dependence of $Y_{1}$ (Fig. 7) is driven by modification of the nonlinear elastic modulus $Y_{1}$ on surface. It is shown in Sec. 3.2 that water adsorption substantially nullifies the bond loss effect and relaxation at the surface atom layer by restoring surface $\mathrm{Zn}-\mathrm{O}$ to bulk coordination. Such surface structure changes sharply decrease the difference in $Y_{0}$ between the surface layer and the interior layers (Fig.4) but increase the gap in $Y_{1}$ between the outmost layer and the inner layers (Fig.8). In particular, the effect of adsorption type on the surface $Y_{1}$ shown in Fig. $8 b$ is found to be consistent with the effect observed on the size-dependence of the overall $Y_{1}$ (Fig. 7).

To summarize water adsorption induced-structural changes at the surface layer largely reduces the significance of the nonlinearity in the surface elasticity or increases the surface $Y_{1}\left(\left|Y_{1}\right|\right.$ decreases), which in turn lead to a higher surface $Y_{1}$ (Fig. 8) and stronger sizedependence of the overall $Y_{1}$ of the NWs (Fig. 7). Thus, when the water adsorption is attached to the NW surface the strain softening of $\mathrm{ZnO} N W s$ turns out to be smaller and the extent of nonlinear elasticity becomes more sensitive to NW diameter change.

In calculating elastic properties of ZnO NWs, inconsistency is found in the literature due to, e.g., different modelling techniques, potential functions and/or theories of elasticity used in analyses. Agreement however is achieved in predicting and understanding the general sizedependence of the elastic properties. The present study therefore is able to get useful insights into the water adoption effect on the size-dependence of the elasticity and reveal its pathway to modify the NW elasticity. 


\section{Conclusions}

The inclusion of non-linear elasticity contribution to strain energy is vital for $\mathrm{ZnO}$ c-axis NW mechanical response prediction. As evident in pristine NWs of $2.2-15 \mathrm{~nm}$, the substantially nonlinear regime (with $R_{S, Y_{1}}>10 \%$ ) is reached at the critical strain $\varepsilon_{c r}$ between 0.05 and 0.056, and requires consideration of at least the third order effective modulus $Y_{o}+Y_{1} \varepsilon$ to adequately describe the strain softening of the NWs. More significantly, the fast convergence of $\varepsilon_{c r}$ toward the asymptotic value of 0.056 at diameter $15 \mathrm{~nm}$ in combination with the failure strain of up to $0.15^{52}$ indicates that non-linearity of elasticity is inherent to these NWs and remains substantial in a wide range of strain.

Upon $1 \mathrm{ML}$ water passivation, the surface $\mathrm{Zn}-\mathrm{O}$ relaxation due to bond loss is significantly reduced by water adsorption due to the restoration of surface $\mathrm{Zn}$ or O to a fully coordinated state. This produces the reduction of the equilibrium c-axis constant on surface relative to the pristine value. More importantly, additional energy is stored during tensile deformation in the water adlayer and the newly established $\mathrm{ZnO}$ bonds on the NW surface. The surface structural changes yield a higher surface linear elastic modulus reducing the difference between the inner and surface values compared to their pristine counterparts. Thus, water adsorption, by suppressing the surface effect, leads to higher linear elastic modulus and a reversed but less pronounced size-dependence trend.

On the surface layer of pristine NWs the negative nonlinear elastic modulus is higher than those of the inner layers, which suggests that the non-linearity or strain softening on the surface atom interaction is less significant and gives rise to the overall nonlinear elastic modulus decreasing with the growing size. $1 \mathrm{ML}$ Water adsorption on the NW surface further reduces the significance of the nonlinearity of the surface atom interaction and thus yields an 
even higher surface nonlinear elastic modulus. This finally enhances the overall nonlinear elastic modulus of the pristine NWs and its size-dependence but narrows down the strain range of the nonlinear elastic response. Thus, strain softening effect achieved for water passivated NWs is substantially lower as compared with that of its pristine counterparts.

\section{Data Availability}

The raw/processed data required to reproduce these findings cannot be shared at this time due to technical or time limitations.

\section{Reference}

(1) Wang, Z. L. Towards Self-Powered Nanosystems: From Nanogenerators to Nanopiezotronics. Advanced Functional Materials 2008, 18 (22), 3553-3567. https://doi.org/10.1002/adfm.200800541.

(2) Yang, X.; Daoud, W. A. Triboelectric and Piezoelectric Effects in a Combined TriboPiezoelectric Nanogenerator Based on an Interfacial ZnO Nanostructure. Advanced Functional Materials 2016, 26 (45), 8194-8201.

https://doi.org/10.1002/adfm.201602529.

(3) Chen, Z.; Wang, Z.; Li, X.; Lin, Y.; Luo, N.; Long, M.; Zhao, N.; Xu, J. Bin. Flexible Piezoelectric-Induced Pressure Sensors for Static Measurements Based on Nanowires/Graphene Heterostructures. ACS Nano 2017, 11 (5), 4507-4513. https://doi.org/10.1021/acsnano.6b08027.

(4) Muralt, P.; Polcawich, R. G.; Trolier-McKinstry, S. Piezoelectric Thin Films for Sensors, Actuators, and Energy Harvesting. MRS Bulletin 2009, 34 (9), 658-664. https://doi.org/10.1557/mrs2009.177.

(5) Wu, W.; Wang, Z. L. Piezotronics and Piezo-Phototronics for Adaptive Electronics and Optoelectronics. Nature Reviews Materials. 2016, p 16031. https://doi.org/10.1038/natrevmats.2016.31.

(6) Wang, Z. L. Piezopotential Gated Nanowire Devices: Piezotronics and PiezoPhototronics. Nano Today. 2010, pp 540-552. https://doi.org/10.1016/j.nantod.2010.10.008.

(7) Yang, R.; Qin, Y.; Li, C.; Zhu, G.; Lin Wang, Z. Converting Biomechanical Energy into Electricity by a Muscle-Movement-Driven Nanogenerator. Nanoletters 2009, 9 (3), 1201-1205. https://doi.org/10.1021/nl803904b. 
(8) Espinosa, H. D.; Bernal, R. a.; Minary-Jolandan, M. A Review of Mechanical and Electromechanical Properties of Piezoelectric Nanowires. Advanced Materials 2012, 24 (34), 4656-4675. https://doi.org/10.1002/adma.201104810.

(9) Wang, S.; Shan, Z.; Huang, H. The Mechanical Properties of Nanowires. Advanced Science 2017, 4 (4), 1600332. https://doi.org/10.1002/advs.201600332.

(10) Park, H. S.; Cai, W.; Espinosa, H. D. Mechanics of Nanowires. MRS Bulletin 2009, 34 (March).

(11) Huang, Y.; Bai, X.; Zhang, Y. In Situ Mechanical Properties of Individual ZnO Nanowires and the Mass Measurement of Nanoparticles. Journal of Physics Condensed Matter 2006, 18, L179. https://doi.org/10.1088/0953-8984/18/15/L03.

(12) Asthana, a; Momeni, K.; Prasad, a; Yap, Y. K.; Yassar, R. S. In Situ Observation of SizeScale Effects on the Mechanical Properties of ZnO Nanowires. Nanotechnology 2011, 22 (26), 265712. https://doi.org/10.1088/0957-4484/22/26/265712.

(13) Chen, C.; Shi, Y.; Zhang, Y.; Zhu, J.; Yan, Y. Size Dependence of Young's Modulus in ZnO Nanowires. Physical Review Letters 2006, 96 (7), 75505.

https://doi.org/10.1103/PhysRevLett.96.075505.

(14) Huang, Y.; Zhang, Y.; Wang, X.; Bai, X.; Gu, Y.; Yan, X.; Liao, Q.; Qi, J.; Liu, J. Size Independence and Doping Dependence of Bending Modulus in ZnO Nanowires. Crystal Growth and Design 2009, 9 (4), 1640-1642. https://doi.org/10.1021/cg800535z.

(15) Wen, B.; Sader, J. E.; Boland, J. J. Mechanical Properties of ZnO Nanowires. Physical Review Letters 2008, 101 (17), 175502.

https://doi.org/10.1103/PhysRevLett.101.175502.

(16) Xu, F.; Qin, Q.; Mishra, A.; Gu, Y.; Zhu, Y. Mechanical Properties of Zno Nanowires under Different Loading Modes. Nano Research 2010, 3 (4), 271-280. https://doi.org/10.1007/s12274-010-1030-4.

(17) Desai, A. V.; Haque, M. A. Mechanical Properties of ZnO Nanowires. Sensors and Actuators, A: Physical 2007, 134 (1), 169-176. https://doi.org/10.1016/j.sna.2006.04.046.

(18) Christiansen, S. H.; Hoffmann, S.; Ballif, C.; Zacharias, M.; Östlund, F.; Michler, J.; Fan, H. J. Fracture Strength and Young's Modulus of ZnO Nanowires. Nanotechnology 2007, 18 (20), 205503. https://doi.org/10.1088/0957-4484/18/20/205503.

(19) Agrawal, R.; Peng, B.; Espinosa, H. D. Experimental-Computational Investigation of ZnO Nanowires Strength and Fracture. Nano letters 2009, 9 (12), 4177-4183. https://doi.org/10.1021/n19023885.

(20) Roy, A.; Mead, J.; Wang, S.; Huang, H. Effects of Surface Defects on the Mechanical Properties of ZnO Nanowires. Scientific Reports 2017, 7 (1), 1-8. https://doi.org/10.1038/s41598-017-09843-5. 
(21) Hoang, M. T.; Yvonnet, J.; Mitrushchenkov, A.; Chambaud, G. First-Principles Based Multiscale Model of Piezoelectric Nanowires with Surface Effects. Journal of Applied Physics 2013, 113 (1), 014309. https://doi.org/10.1063/1.4773333.

(22) Wang, X.; Gu, Y.; Sun, X.; Wang, H.; Zhang, Y. Third-Order Elastic Constants of ZnO and Size Effect in ZnO Nanowires. Journal of Applied Physics 2014, 115 (21), 0-8. https://doi.org/10.1063/1.4881775.

(23) Yvonnet, J.; Mitrushchenkov, A.; Chambaud, G.; He, Q. C.; Gu, S. T. Characterization of Surface and Nonlinear Elasticity in Wurtzite ZnO Nanowires. Journal of Applied Physics 2012, 111 (12). https://doi.org/10.1063/1.4729545.

(24) Agrawal, R.; Paci, J. T.; Espinosa, H. D. Large-Scale Density Functional Theory Investigation of Failure Modes in ZnO Nanowires. Nano Letters 2010, 10 (9), 34323438. https://doi.org/10.1021/nl1014926.

(25) Agrawal, R.; Peng, B.; Gdoutos, E. E.; Espinosa, H. D. Elasticity Size Effects in ZnO Nanowires-A Combined Experimental-Computational Approach. Nano Letters 2008, 8 (11), 3668-3674. https://doi.org/10.1021/nl801724b.

(26) Bernal, R. A.; Agrawal, R.; Peng, B.; Bertness, K. A.; Sanford, N. A.; Davydov, A. V.; Espinosa, H. D. Effect of Growth Orientation and Diameter on the Elasticity of GaN Nanowires. A Combined in Situ TEM and Atomistic Modeling Investigation. Nano Letters 2011, 11 (2), 548-555. https://doi.org/10.1021/nl103450e.

(27) Wang, R. J.; Wang, C. Y.; Feng, Y. T. Effective Geometric Size and Bond-Loss Effect in Nanoelasticity of GaN Nanowires. International Journal of Mechanical Sciences 2017, 130, 267-273. https://doi.org/10.1016/j.ijmecsci.2017.06.026.

(28) Meyer, B.; Rabaa, H.; Marx, D. Water Adsorption on ZnO(1010): From Single Molecules to Partially Dissociated Monolayers. Physical Chemistry Chemical Physics 2006, 8 (13), 1513-1520. https://doi.org/10.1039/b515604a.

(29) Meyer, B.; Marx, D.; Dulub, O.; Diebold, U.; Kunat, M.; Langenberg, D.; Wöll, C. Partial Dissociation of Water Leads to Stable Superstructures on the Surface of Zinc Oxide. Angewandte Chemie - International Edition 2004, 43 (48), 6642-6645. https://doi.org/10.1002/anie.200461696.

(30) Raymand, D.; van Duin, A. C. T.; Spångberg, D.; Goddard, W. A.; Hermansson, K. Water Adsorption on Stepped ZnO Surfaces from MD Simulation. Surface Science 2010, 604 (9-10), 741-752. https://doi.org/10.1016/j.susc.2009.12.012.

(31) Kenmoe, S.; Ulrich Biedermann, P. Water Aggregation and Dissociation on the ZnO(1010) Surface. Physical Chemistry Chemical Physics 2017, 19 (2), 1466-1486. https://doi.org/10.1039/c6cp07516a.

(32) Newberg, J. T.; Goodwin, C.; Arble, C.; Khalifa, Y.; Boscoboinik, J. A.; Rani, S. $\mathrm{ZnO}(1010)$ Surface Hydroxylation under Ambient Water Vapor. Journal of Physical Chemistry B 2018, 122 (2), 472-478. https://doi.org/10.1021/acs.jpcb.7b03335. 
(33) Yang, Y.; Wang, G.; Li, X. Water Molecule-Induced Stiffening in ZnO Nanobelts. Nano Letters 2011, 11 (7), 2845-2848. https://doi.org/10.1021/nl201237x.

(34) Zhang, J.; Zhou, J. Humidity-Dependent Piezopotential Properties of Zinc Oxide Nanowires: Insights from Atomic-Scale Modelling. Nano Energy 2018, 50, 298-307. https://doi.org/10.1016/j.nanoen.2018.05.054.

(35) Ding, Y.; Zhang, F.; Wang, Z. L. Deriving the Three-Dimensional Structure of ZnO Nanowires/Nanobelts by Scanning Transmission Electron Microscope Tomography. Nano Research 2013, 6 (4), 253-262. https://doi.org/10.1007/s12274-013-0301-2.

(36) Yang, K.; She, G. W.; Wang, H.; Ou, X. M.; Zhang, X. H.; Lee, C. S.; Lee, S. T. ZnO Nanotube Arrays as Biosensors for Glucose. Journal of Physical Chemistry C 2009, 113 (47), 20169-20172. https://doi.org/10.1021/jp901894j.

(37) Agrawal, R.; Peng, B.; Gdoutos, E. E.; Espinosa, H. D. Elasticity Size Effects in ZnO Nanowires-A Combined Experimental- Computational Approach. Nano Letters 2008, 8 (11), 3668-3674. https://doi.org/10.1021/nl801724b.

(38) Dulub, O.; Meyer, B.; Diebold, U. Observation of the Dynamical Change in a Water Monolayer Adsorbed on a ZnO Surface. Physical Review Letters 2005, 95 (13), 1-4. https://doi.org/10.1103/PhysRevLett.95.136101.

(39) Wang, Y.; Muhler, M.; Wöll, C. Spectroscopic Evidence for the Partial Dissociation of H2O on ZnO(1010). Physical Chemistry Chemical Physics 2006, 8, 1512-1524. https://doi.org/10.1039/b515489h.

(40) Xu, H.; Zhang, R. Q.; Tong, S. Y. Interaction of O2, H2 O, N2, and O 3 with Stoichiometric and Reduced ZnO (10 10) Surface. Physical Review B - Condensed Matter and Materials Physics 2010, 82 (15), 155326.

https://doi.org/10.1103/PhysRevB.82.155326.

(41) Calzolari, A.; Catellani, A. Water Adsorption on Nonpolar ZnO(1010) Surface: A Microscopic Understanding. Journal of Physical Chemistry C 2009, 113 (7), 28962902. https://doi.org/10.1021/jp808704d.

(42) Hellström, M.; Jorner, K.; Bryngelsson, M.; Huber, S. E.; Kullgren, J.; Frauenheim, T.; Broqvist, P. An SCC-DFTB Repulsive Potential for Various ZnO Polymorphs and the ZnO-Water System. Journal of Physical Chemistry C 2013, 117 (33), 17004-17015. https://doi.org/10.1021/jp404095x.

(43) Ewing, G. E. Ambient Thin Film Water on Insulator Surfaces. Chemical Reviews 2006, 106 (4), 1511-1526. https://doi.org/10.1021/cr040369x.

(44) Henderson, M. A. The Interaction of Water with Solid Surfaces: Fundamental Aspects Revisited. Surf. Sci. Rep. 2002, 46 (1-8), 1-308. https://doi.org/10.1016/S01675729(01)00020-6.

(45) Rubasinghege, G.; Grassian, V. H. Role(s) of Adsorbed Water in the Surface Chemistry of Environmental Interfaces. Chemical Communications 2013, 49, 3071-3094. 
https://doi.org/10.1039/c3cc38872g.

(46) Yan, Y.; Al-Jassim, M. M. Structure and Energetics of Water Adsorbed on the $\mathrm{ZnO}(1010)$ Surface. Physical Review B - Condensed Matter and Materials Physics 2005, 72 (23), 1-6. https://doi.org/10.1103/PhysRevB.72.235406.

(47) Van Duin, A. C. T.; Dasgupta, S.; Lorant, F.; Goddard, W. A. ReaxFF: A Reactive Force Field for Hydrocarbons. Journal of Physical Chemistry A 2001, 105 (41), 9396-9409. https://doi.org/10.1021/jp004368u.

(48) Raymand, D.; van Duin, A. C. T.; Baudin, M.; Hermansson, K. A Reactive Force Field (ReaxFF) for Zinc Oxide. Surface Science 2008, 602 (5), 1020-1031. https://doi.org/10.1016/j.susc.2007.12.023.

(49) Raymand, D.; Van Duin, A. C. T.; Goddard, W. A.; Hermansson, K.; Spångberg, D. Hydroxylation Structure and Proton Transfer Reactivity at the Zinc Oxide-Water Interface. Journal of Physical Chemistry C 2011. https://doi.org/10.1021/jp106144p.

(50) Plimpton, S. Fast Parallel Algorithms for Short - Range Molecular Dynamics. Journal of Computational Physics 1995, 117 (June 1994), 1-19.

https://doi.org/10.1006/jcph.1995.1039.

(51) Hu, H.; Ji, H. F.; Sun, Y. The Effect of Oxygen Vacancies on Water Wettability of a ZnO Surface. Physical Chemistry Chemical Physics 2013, 15 (39), 16557-16565. https://doi.org/10.1039/c3cp51848e.

(52) Wang, J.; Shen, Y.; Song, F.; Ke, F.; Liao, X. On the Wurtzite to Tetragonal Phase Transformation in ZnO Nanowires. Nanotechnology 2017, 28 (100), 165705. https://doi.org/10.1088/1361-6528/aa6566.

(53) Zhou, L. G.; Huang, H. Are Surfaces Elastically Softer or Stiffer? Applied Physics Letters 2004, 84 (11), 1940-1942. https://doi.org/10.1063/1.1682698.

(54) Zhang, L.; Huang, H. Young's Moduli of ZnO Nanoplates: Ab Initio Determinations. Applied Physics Letters 2006, 89 (18), 9-12. https://doi.org/10.1063/1.2374856.

(55) He, M.-R.; Shi, Y.; Zhou, W.; Chen, J. W.; Yan, Y. J.; Zhu, J. Diameter Dependence of Modulus in Zinc Oxide Nanowires and the Effect of Loading Mode: In Situ Experiments and Universal Core-Shell Approach. Applied Physics Letters 2009, 95 (9), 091912. https://doi.org/10.1063/1.3205102. 